\title{
On Chaotic Subthreshold Oscillations in a Simple Neuronal Model
}

\author{
M. Zaks * \\ Institute of Physics, Humboldt University of Berlin, D-12489, Germany
}

\begin{abstract}
In a simple FitzHugh-Nagumo neuronal model with one fast and two slow variables, a sequence of period-doubling bifurcations for small-scale oscillations precedes the transition into the spiking regime. For a wide range of values of the timescale separation parameter, this scenario is recovered numerically. Its relation to the singularly perturbed integrable system is discussed.
\end{abstract}

Key words: slow-fast equations, period-doubling bifurcation

AMS subject classification: $92 \mathrm{C} 20,37 \mathrm{G} 15,34 \mathrm{D} 15$

\section{Introduction}

Excitability is one of the central notions in the mathematical theory of neural cells. In certain classes of cells, after application of an external electric pulse, the transmembrane voltage performs a large and fast excursion (an action potential), before returning to the state of rest; for details see e.g. the textbook [10]. Sequences of such action potentials play important role in the transmission of information between the cells. Mathematical studies of generation and propagation of signals across the networks of neurons go back to the pioneering works of Hodgkin and Huxley (see e.g. [10] for references and detailed discussions). In the last two decades a variety of new models and approaches have strongly facilitated our understanding of neural processes.

For many neurons action potentials occupy only a minor proportion of time. In the intervals between the consecutive action potentials (so-called interspike intervals, ISI), the real in vivo neurons neither are quiescent nor display purely monotonic evolution to the state of equilibrium: instead,

*E-mail: zaks@physik.hu-berlin.de 
they perform small-scale oscillations. Sometimes such oscillations (called "subthreshold oscillations" since their amplitude is insufficient for initiation of a spike) have complicated temporal patterns. Commonly, the irregularity of subthreshold oscillations (as well as, sometimes, their very existence, see. e.g. [16]) is ascribed to the influence of omnipresent stochastic fluctuations which disturb the system. Below we demonstrate that irregular subthreshold oscillations can emerge in the deterministic setup. Previously, such small-scale chaotic oscillations were reported for collective variables in models of large ensembles of neurons [18]. Here we show that onset of chaos may precede the transition to the spiking state in a simple model of an isolated neuron. Aiming at transparency, we deliberately choose the oversimplified setup with the lowest possible dimension of the phase space and the small number of parameters. The model is a FitzHugh-Nagumo-like set of three ODEs which includes one fast membrane potential and two slow gating variables. In the phase space, the attractor which corresponds to chaotic subthreshold oscillations, is located near the fold of the two-dimensional surface of slow motions. Onset of spiking regime is interpreted as a kind of "canard transition" which, in contrast to the conventional case, concerns not a single oneparameter family of periodic orbits but the whole attracting set. In Section 2. the model equations are introduced, and the basic bifurcation scenarios are described. In Section 3. we compute an accurate approximation of the slow surface, and characterize the details of dynamics near its fold. Finally, in Section 4. the case of small and vanishing values of the timescale separation coefficient $\varepsilon$ is considered. It is shown that decrease of $\varepsilon$ leads to crossover between two universality classes of the period-doubling scenario, and that in the limit $\varepsilon \rightarrow 0$ the system becomes conservative with two explicit integrals.

\section{Model equations: properties and bifurcations}

Below we consider a simple toy set of ODEs which models an interaction of membrane potential with two gating variables. The typical $N$-shaped self-action of the potential $X$ is mimicked by the cubic nonlinearity. The gating variables $Y$ and $Z$ do not interact; their respective evolution equations, as well as their inhibiting action upon $X$, are assumed to be linear. Under these provisions, the most general form of the dynamical equations is:

$$
\begin{aligned}
\dot{X} & =\alpha_{0}+\alpha_{1} X+\alpha_{2} X^{2}-\alpha_{3} X^{3}-\delta_{1} Y-\delta_{2} Z \\
\dot{Y} & =\beta_{0}+\beta_{1} X-\beta_{2} Y \\
\dot{Z} & =\gamma_{0}+\gamma_{1} X-\gamma_{2} Z
\end{aligned}
$$

where, among the coefficients, $\alpha_{1}, \alpha_{3}, \delta_{1,2}, \beta_{1}$ and $\gamma_{1}$ are considered to be positive; $\beta_{2}$ and $\gamma_{2}$ are non-negative, and the sign of the rest can be arbitrary. A linear transformation - shift and stretching of each variable, accompanied by rescaling of time units - casts these equations into the FitzHugh-Nagumo-like shape [10]: 


$$
\begin{aligned}
\varepsilon \dot{x} & =x-x^{3} / 3-d y-z \\
\dot{y} & =a+x-b y \\
\dot{z} & =a+x-c z
\end{aligned}
$$

In these equations, $a$ is an "excitability parameter", and the coefficients $\varepsilon, d$ are positive. Each of the inhibitors $y$ and $z$ is driven by the instantaneous concentration $x$ of the activator, and is, additionally, linearly damped, with non-negative respective "damping rates" $b$ and $c$.

Here, we do not aim at the full exploration of the 5-dimensional parameter space of Eq.(2.2). The equations are invariant with respect to the simultaneous change of sign of $x, y, z$ and $a$; therefore the bifurcation diagram is even with respect to $a$, and it is sufficient to consider, say, the range $a>0$. Furthermore, in accordance with physiological background, we concentrate on the region $\varepsilon \ll 1$. This allows to separate the timescales: $x$ is the fast variable, whereas two gating variables $y$ and $z$ are slow. Finally, we restrict us to the "proportional" case $d=1$; in terms of original parameters of $\operatorname{Eqs}(2.1)$, this requires $\beta_{1} / \gamma_{1}=\delta_{2} / \delta_{1}$.

Equations, reducible to (2.1) are known as "generalized" or "modified" FitzHugh-Nagumo equations: the earliest references, besides the unpublished studies of FitzHugh \& Rinzel from 1976, are [7] and [12].

Below, we will mostly treat $a$ as the bifurcation parameter under fixed values of $\varepsilon, b$ and $c$. For

$$
a^{2}>\frac{4(b c-b-c)^{3}}{9 b c(b+c)^{2}}
$$

equations (2.2) possess a single steady solution, otherwise three steady states are present in the phase space. We restrict the analysis to the former situation: the parameter range $b c<b+c$ in which, irrespective of $a$, the equilibrium state is unique.

For sufficiently large values of $|a|$ the equilibrium is stable. Decrease of $|a|$ lets two complex eigenvalues in the Jacobian of this equilibrium cross into the right half-plane: this is the AndronovHopf bifurcation which occurs (recall the smallness of $\varepsilon$ ) at

$$
|a|=a_{H}=1-\frac{2 b c}{3(b+c)}-\frac{1}{4}(b+c) \varepsilon-\frac{(b+c)(6 b c+(b+c)(1-2 b-2 c))}{32} \varepsilon^{2}+O\left(\varepsilon^{3}\right)
$$

Immediately after the supercritical Andronov-Hopf bifurcation at $a_{H}$ the amplitude of the newborn stable periodic orbit is small and lies below the threshold of spiking. In contrast, far away from that bifurcation (at $|a| \ll a_{H}$ ), the system exhibits large-scale periodic oscillations: continuous spiking. Different scenarios of transition between these two states are possible. The most straightforward one is illustrated by the case $b=c$ : here, the plane $y=z$ in the phase space is globally attracting, and dynamics upon it obeys the second order FitzHugh-Nagumo equation [10].

As visualized in Fig. 1, the minute parameter difference causes drastic changes in the shape and size of the limit cycle. Transformation of the orbit occurs within the narrow parameter interval $\sim \exp (-1 / \varepsilon)[4,6,1]$, and is known as the "canard explosion". The wavetrain of the spiking state, 

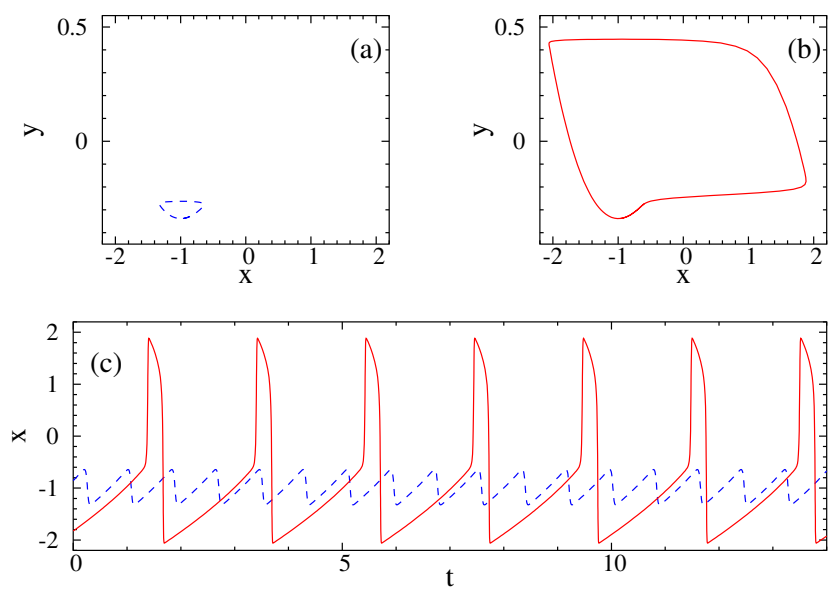

Figure 1: (Color Online). Canard explosion at $\varepsilon=10^{-2}, b=c=0.1$. a),b) projections of phase portraits; c) temporal profile of the oscillations. Dashed curves: $a=0.96387830$; solid curves: $a=0.96387829$. For comparison: $a_{H}=0.9994489$

shown in the bottom panel of Fig. 1, has a typical shape of the relaxation oscillation: segments of relatively slow evolution are separated by short spikes with the strong variation of the variable. This is a consequence of the presence in the phase space of the folded two-dimensional "slow surface" (of which only the "slow curve" - its intersection with the attracting plane $y=z-$ is of relevance). In the course of time, epochs of slow motion along attracting parts of the slow surface alternate with rapid flights across the phase space (shown by nearly horizontal orbit segments in the top right panel). Canard explosion is caused by the passage of trajectories along unstable parts of the slow surface.

In the two-dimensional dynamics of FitzHugh-Nagumo equations there is no room for more complicated states than in Fig. 1. At $b \neq c$, in the really three-dimensional setup, more elaborate phase trajectories are allowed. An elemental oscillation consists of the following stages:

a) the system moves along the attracting part of the slow surface,

b) it crosses the fold,

c) it continues the motion along the repelling part,

d) finally it departs from the slow surface and bounces back to the attracting part.

Unlike the case $b=c$, dynamics on the slow surface can effectively become two-dimensional, and subsequent returns onto this surface should not necessarily hit the same point. As a result, the initial periodic state may lose stability already before the canard transition. An example is shown in Fig. 2: subthreshold oscillations exhibit a sequence of period-doubling bifurcations, and the small-scale chaotic attractor arises. Notably, in the region near the fold (the bottom part of the plots) the distance between the different turns of the orbit is hardly graphically resolvable, in contrast to the "flights" from the repelling part of the slow surface to the attracting one, which occur at different "heights". Existence of the chaotic state confirms that subthreshold oscillations can get irregular without fluctuations!

Further decrease of $a$ produces qualitative changes in the phase portrait. Comparison of the 

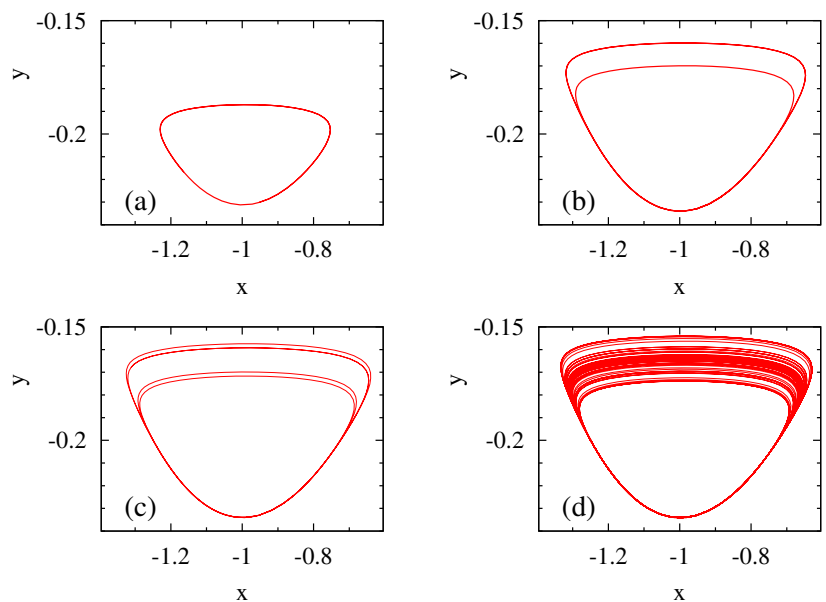

Figure 2: (Color Online). Subthreshold oscillations: phase portraits for at $\varepsilon=10^{-2}, b=0.2, c=0.1$. a) $a=0.9525$; b) $a=0.9523060$; c) $a=0.9523035$; d) $a=0.9523013$.

oscillation amplitudes in the two top panels of Fig. 3 indicates to the transition from subthreshold oscillations to the spiking regime: a minor parameter variation invokes a tremendous growth of the amplitude. Apparently, this is a variant of the canard explosion which concerns, however, not the individual periodic orbit, but the whole attractor $[18,15]$.
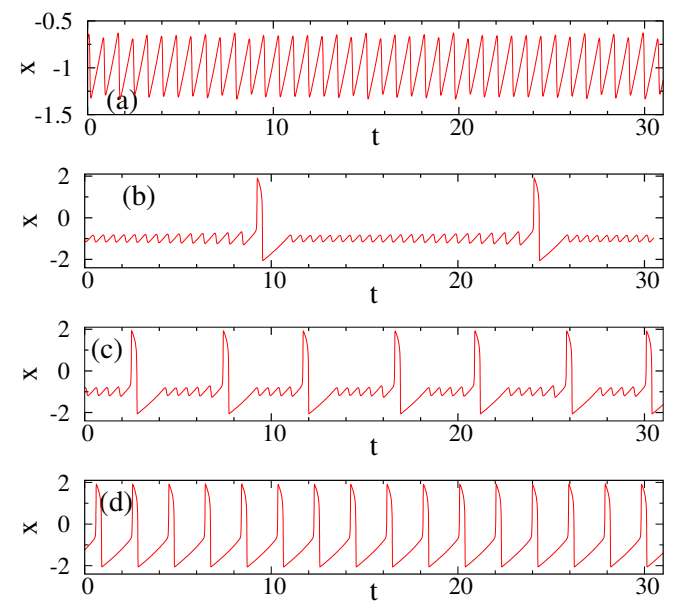

Figure 3: (Color Online). Oscillatory states at $\varepsilon=10^{-2}, b=0.2, c=0.1$. a) chaotic subthreshold oscillations, $a=0.9523013$; b) $a=0.9523005$; c) $a=0.9510$; d) $a=0.9486$.

Initially, the spikes are seldom: they are interspersed with numerous subthreshold oscillations (Fig. 3b). As $a$ is further increased, the interspike intervals become shorter (Fig. 3c) until, finally, the subthreshold oscillations disappear completely, and the system displays uninterrupted spiking.

Regimes in which the large oscillations alternate with small ones are known as "mixed-mode oscillations" [11]; one of the mechanisms of their onset is related to canards in systems with two 
and more slow variables $[11,2,14]$. Mixed-mode oscillations are believed to stand behind a variety of phenomena in neuronal dynamics and in physical chemistry (see e.g. the Focus Issue [3]). In Fig. 4 projections of phase portraits for different stages of the evolution of the spiking state are shown. Note that the whole span of chaotic subthreshold oscillations in Fig.2d corresponds to the small "knot" in the lower left corner of the portrait in Fig.4a.
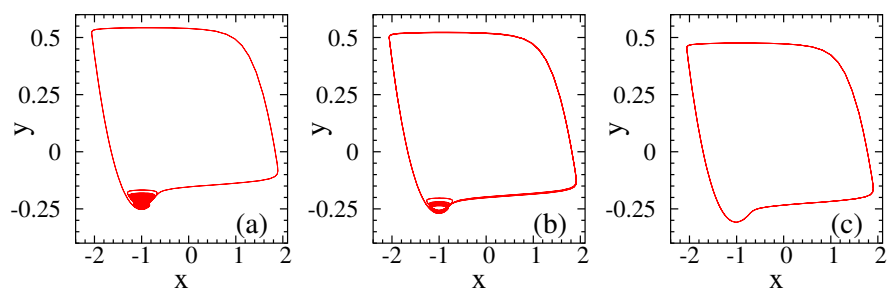

Figure 4: (Color Online). Phase portraits in the spiking state at $\varepsilon=10^{-2}, b=0.2, c=0.1$. a) $a=0.9523005$; b) $a=0.9510$; c) $a=0.9486$.

\section{Surface of slow motions}

Transition from subthreshold oscillations to spiking, as well as the form of the spiking wavetrains depend on the shape and position of the two-dimensional surface which corresponds to slow motions in the phase space of Eq.(2.2). In this Section, we compute an approximation to this slow surface and briefly characterize dynamics upon it. For the sake of shortening the lengthy expressions, here the computations are performed for the case $b=0$, so that $a_{H}=1+O(\varepsilon)$.

\subsection{Location of the slow surface}

In terms of $u \equiv y+z$ the equations turn into

$$
\begin{aligned}
\varepsilon \dot{x} & =x-\frac{x^{3}}{3}-u \\
\dot{u} & =2(x+a)-c z \\
\dot{z} & =x+a-c z .
\end{aligned}
$$

In the zeroth order in $\varepsilon$, the slow surface is obtained by setting $\varepsilon=0$ in the first equation, and turning it thereby into the algebraic relation:

$$
u=u_{0}=x-x^{3} / 3
$$

In the phase space the surface $u_{0}$ has a fold at $x=-1$. The steady state lies upon $u_{0}$; when the value of $a$ is varied, position of the equilibrium moves, and at $a=a_{H}$ it arrives at the fold. 
We are interested in the local description: neighborhood of the fold in the phase space, and the parameter region around $a_{H}$. A convenient coordinate close to the fold is $\xi \equiv x+1$. The first order correction to $u_{0}$ for the slow surface is:

$$
u=u_{0}-\varepsilon\left(\kappa_{0}-\kappa_{1} \xi\right)
$$

with yet unknown $\kappa_{0}$ and $\kappa_{1}$. Evolution equation for $z$ is rewritten as $\dot{z}=\xi+G-c z$ with $G \equiv a-1$; according to numerical data, in the parameter range of interest $\frac{G}{\varepsilon}=O(1)$, therefore $G$ has an order of smallness of $\varepsilon$. Now,

$$
\dot{\xi}=\dot{x}=\left(u-u_{0}\right) / \varepsilon=\kappa_{0}-\kappa_{1} \xi,
$$

and the equation for $d z / d \xi$ can be integrated explicitly. Expansion of $z(\xi)$ up to quadratic terms yields

$$
z(\xi)=C_{I}+\frac{G\left(c-\kappa_{1}\right)-\kappa_{0}}{c\left(c-\kappa_{1}\right)}+\left(\frac{1}{c-\kappa_{1}}-\frac{c C_{I}}{\kappa_{0}}\right) \xi+\frac{c C_{I}\left(c-\kappa_{1}\right)}{2 \kappa_{0}^{2}} \xi^{2}
$$

with integration constant $C_{I}$. Substituting this into

$$
2(\xi+G)-c z=\dot{u}=\dot{\xi}\left(2 \xi-\xi^{2}+\varepsilon \kappa_{1}\right)=\left(\kappa_{0}-\kappa_{1} \xi\right)\left(2 \xi-\xi^{2}+\varepsilon \kappa_{1}\right)
$$

and equating the coefficients at $\xi^{0}, \xi^{1}$ and $\xi^{2}$ results in three equations for $\kappa_{0}, \kappa_{1}$ and $C_{I}$. Up to the higher order terms, this gives

$$
\kappa_{0}=1+\frac{(2-c)(2+3 c) \varepsilon+16 G c}{32} \quad \text { and } \quad \kappa_{1}=\frac{c-2}{4}\left(1+\frac{(2-c)(2+3 c) \varepsilon}{32}+\frac{G c}{4}\right) .
$$

Besides,

$$
C_{I}=\frac{4}{c(2+3 c)}-\varepsilon \frac{(c-2)(2+5 c)}{4 c(2+3 c)}+G \frac{2\left(2+7 c+8 c^{2}\right)}{b(2+3 c)^{2}}
$$

so that

$$
c z=2 G+\frac{2-c}{4} \varepsilon+c\left(\frac{c-2}{4} \varepsilon-G\right) \xi+\left(\frac{c}{2}-\frac{c(c-2)(2+3 c)}{64} \varepsilon+\frac{c(2+c)}{8} G\right) \xi^{2}
$$

Retaining the leading terms in these expressions (recall $G \sim \varepsilon$ ) we arrive at the approximation for the slow surface. In terms of original variables, it reads

$$
u_{\mathrm{sl}}=y+z=x-\frac{x^{3}}{3}-\varepsilon\left(1+\frac{2-c}{4}(x+1)\right)
$$

The accuracy of this estimate is confirmed graphically in Fig. 5 for the periodic orbit close to $a_{H}$. As seen on the bottom left panel, the orbit as a whole is located "at the fold" of the slow surface. The top panel shows that most of the period is spent in the immediate neighborhood of $u_{s}$. The bottom right panel indicates that the distance to $u_{s}$ during this passage is definitely smaller than $\varepsilon$ (in fact, it is of the order $\varepsilon^{2}$ ). 

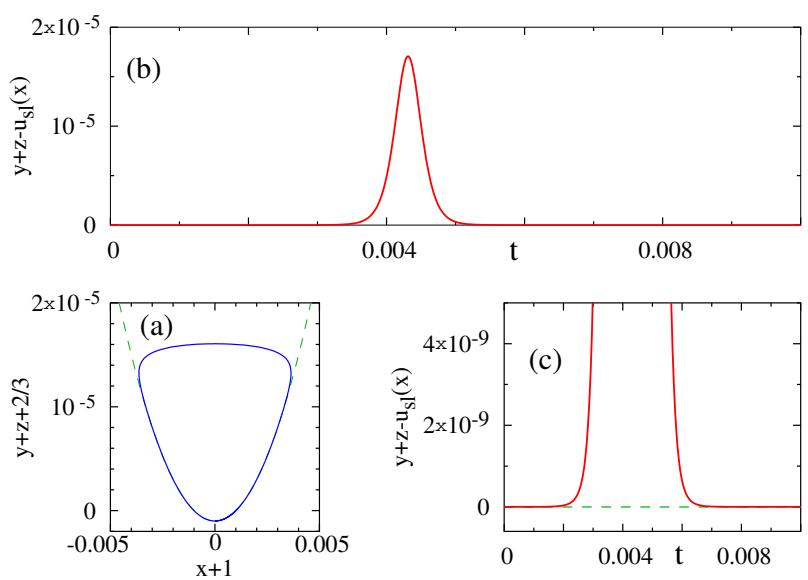

Figure 5: (Color Online). Dynamics near the slow surface $u_{s} . \quad a=0.9999982719, b=0, c=0.1$, $\varepsilon=10^{-6}$. (a) solid line: projection of the phase project, dashed line: slow surface; (b) temporal evolution of the distance from $u_{s}$, as given by Eq.(3.3); (c) blowup of the bottom part of (b).

\subsection{Dynamics on the slow surface}

It is convenient to parameterize the slow surface by the coordinates $\xi$ and $z$. Evolution equations on the slow surface are

$$
\begin{aligned}
\dot{\xi} & =\frac{2(\xi+G)-c z}{2 \xi-\xi^{2}+\varepsilon \kappa_{1}} \\
\dot{z} & =\xi+G-c z
\end{aligned}
$$

In this coordinates the fold which separates the attracting part of the slow surface from the repelling one, is located at $\xi=\xi_{0}=1-\sqrt{1+\varepsilon \kappa_{1}}$. The r.h.s of the first equation possesses a singularity at the fold. To get rid of it, we introduce the coordinate-dependent reparameterization of time: the time unit is multiplied by $\xi^{2}-2 \xi-\varepsilon \kappa_{1}$. Note that this reparameterization reverses the time direction on one side of the fold.

After desingularization, dynamics on the slow surface is governed by

$$
\begin{aligned}
& \xi^{\prime}=c z-2(\xi+G) \\
& z^{\prime}=\left(\xi^{2}-2 \xi-\varepsilon \kappa_{1}\right)(\xi+G-c z)
\end{aligned}
$$

where the prime denotes differentiation with respect to the "new" time.

Along with the usual equilibrium (located at $\xi=-G, z=0$ ), the desingularized equations (3.5) possess an additional one: the so-called "folded equilibrium" [1] which sits exactly on the fold. Its coordinates are:

$$
\xi=\xi_{0} \approx-\frac{\varepsilon \kappa_{1}}{2}, z_{0}=\frac{2\left(\xi_{0}+G\right)}{c} \approx \frac{2 G-\varepsilon \kappa_{1}}{c} .
$$

Eigenvalues of Jacobian at this fixed point are (leading terms) $\lambda_{1} \approx c\left(G-\varepsilon \kappa_{1} / 2\right)$ and $\lambda_{2} \approx-2$. 
For $G>G_{0}=\varepsilon \kappa_{1} / 2$ the usual equilibrium lies on the attracting part of the slow surface and is a stable node, whereas the folded equilibrium is a saddle-point ("folded saddle"). At $G=$ $G_{0}$ the transcritical bifurcation takes place: two fixed points merge and exchange stability. For $G<G_{0}$ the usual equilibrium is a saddle and lies on the repelling part of the slow surface. The folded equilibrium becomes a node. According to [2, 17], one of the reasons for the mixed-mode oscillations may lie in the complicated global topology of the invariant manifolds of the folded node. Slopes of eigenvectors of its Jacobian matrix are $c z_{0} / \lambda_{1} \approx 2 / c$ and $c z_{0} / \lambda_{2} \approx \varepsilon \kappa_{1} / 2-G$, respectively. The first eigenvector corresponds (in terminology of [2]) to the weak canard, whereas the second one which is almost perpendicular to the fold line, corresponds to the strong canard.

\section{Case of small and vanishing $\varepsilon$.}

\subsection{Transformation of bifurcation scenario}

According to our observations, the sequence of period-doubling bifurcations for subthreshold oscillatory states occurs not only under "moderate" (of the order of $10^{-3} \div 10^{-1}$ ) but also under rather small values of $\varepsilon$ : the first three bifurcations from this scenario were numerically detected for $\varepsilon$ as low as $5 \times 10^{-13}$. Decrease of $\varepsilon$ is accompanied by decrease of the period and the amplitude of the oscillations: according to numerical data, they decay as $\sim \sqrt{\varepsilon}$ (with logarithmic corrections). Keeping in mind that the difference between the minimal and maximal values of $x$ in the spiking state approximately equals 4 and is practically independent of $\varepsilon$, this implies that the "canard explosion" of the attractor for small values of $\varepsilon$ increases its size by several orders of magnitude . $^{\dagger}$

In Fig. 6 different stages of evolution of the small-scale oscillations at $\varepsilon=10^{-5}$ are presented. Phase portraits are strongly reminiscent of Fig.2; it is only the scale that differs.
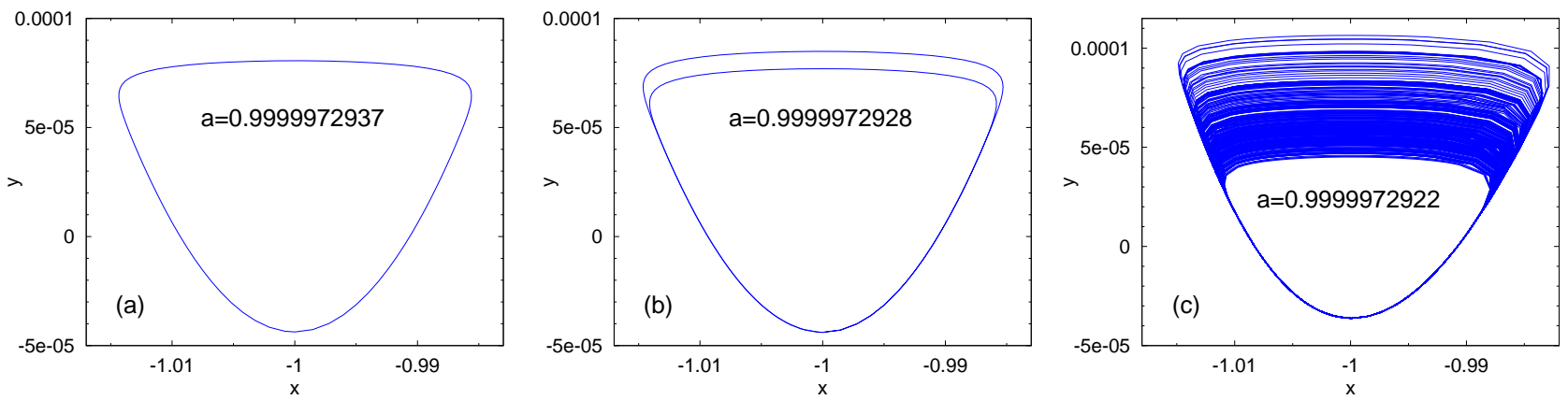

Figure 6: (Color Online). Phase portraits of subthreshold oscillations at $\varepsilon=10^{-5}, b=0, c=1$. a): simple periodic state; b) periodic state after the period-doubling; c) small-scale chaos.

There is, however, another important difference. Under moderate $\varepsilon$, dynamics in the immediate

\footnotetext{
${ }^{\dagger}$ It should be noted, that at low values of $\varepsilon$ the transition is a hysteretic one: the spiking state can coexist with irregular subthreshold oscillations.
} 
vicinity of subthreshold periodic orbits is distinctly dissipative: there is a noticeable contraction along one of the directions in the phase space. As $\varepsilon$ gets smaller, the contraction weakens, and signatures of measure-preserving dynamics evolve. Quantitatively, this evolution is measured by the determinant of the Jacobian matrix of the Poincaré mapping. We have computed this determinant at the fixed-point of the mapping for the value of $a$ which corresponds to the first period-doubling bifurcation. It has turned out that the determinant monotonically grows from 0.42616 at $\varepsilon=0.01$ to 0.999939 at $\varepsilon=10^{-12}$. The latter value means that - at least, sufficiently close to the periodic orbit - the two-dimensional Poincaré mapping nearly preserves area.

Weakening of dissipative properties manifests itself in the characteristics of the bifurcation sequence. From the point of view of renormalization, period-doubling scenarios in dissipative and in measure-preserving systems belong to different classes of universality, with differing quantitative characteristics of the scenarios: convergence rate, scaling factors etc. In the Table below, we estimate the convergence rate of scenario

$$
\delta_{n}=\frac{a_{n+1}-a_{n}}{a_{n+2}-a_{n+1}}
$$

for six first period-doubling bifurcations. Here $a_{n}$ is the value of $a$ which corresponds to the destabilization of the orbit with $2^{n-1}$ turns in the phase space.

\begin{tabular}{|c|c|c|c|c|}
\hline$\varepsilon$ & $\delta_{1}$ & $\delta_{2}$ & $\delta_{3}$ & $\delta_{4}$ \\
\hline \hline $10^{-2}$ & 6.83758 & 4.94214 & 4.68156 & 4.66850 \\
$10^{-3}$ & 8.69566 & 6.33080 & 4.98852 & 4.67011 \\
$10^{-4}$ & 9.56116 & 8.04246 & 6.65841 & 5.18980 \\
$10^{-6}$ & 9.83151 & 8.78821 & 8.64708 & 8.38724 \\
$10^{-8}$ & 9.86815 & 8.81004 & 8.73397 & 8.71522 \\
$10^{-10}$ & 9.89000 & 8.81033 & 8.73596 & 8.72227 \\
$2.3 \times 10^{-11}$ & 9.89563 & 8.81030 & 8.73604 & 8.72236 \\
\hline
\end{tabular}

The data for $\varepsilon \geq 10^{-3}$ indicate straightforward convergence to the universal Feigenbaum constant $\delta_{F}=4.669201 \ldots$, valid for the dissipative systems [8]. For the lower values of $\varepsilon$, however, settling at $\delta_{F}$ is apparently preceded by the intermediate stage, at which the bifurcation rate approaches the value $\delta_{\mathrm{ap}}=8.721097 .$. , universal for area-preserving mappings [9, 5]. This crossover behavior is typical for systems with weak dissipation [19]; duration of the intermediate asymptotics grows with the decrease of $\varepsilon$. 


\subsection{Vanishing $\varepsilon$ : conservative limit.}

In order to analyze dynamics in the limit $\varepsilon \rightarrow 0$, we rescale the independent and dependent variables. The new set: $\tau, X, U$ and $V$ is introduced through relations:

$$
\begin{aligned}
t & =\tau \sqrt{\varepsilon}, \quad x=\sqrt{\varepsilon}(X-1) \\
y & =\frac{\varepsilon}{2}\left(U+V+\frac{2}{3}-\frac{2 \chi_{1}}{3 \chi_{2}}\right) \\
z & =\frac{\varepsilon}{2}\left(U-V+\frac{2}{3}+\frac{2 \chi_{1}}{3 \chi_{2}}\right)
\end{aligned}
$$

with $\chi_{1}=(b+c) / 2$ and $\chi_{2}=(b-c) / 2$.

In terms of new variables the system (2.2) turns into

$$
\begin{aligned}
d X / d \tau & =X^{2}-U-\frac{\sqrt{\varepsilon}}{3} X^{3} \\
d U / d \tau & =2 X-\sqrt{\varepsilon}\left(\chi_{1} U+\chi_{2} V\right)+\sqrt{\varepsilon} A \\
d V / d \tau & =-\sqrt{\varepsilon}\left(\chi_{2} U+\chi_{1} V\right)
\end{aligned}
$$

with

$$
A \equiv \frac{2}{\varepsilon}\left(a-1+\frac{2 b c}{3(b+c)}\right) .
$$

In general, the new parameter $A$ may diverge in the limit $\varepsilon \rightarrow 0$. However, according to the numerical evidence, in the range of $a$ which corresponds to the period-doubling scenario, the difference $\left(a-1+\frac{2}{3} b c /(b+c)\right)$ is proportional to $\varepsilon$ (cf. Fig.7), which implies that $A$ remains finite ${ }^{\ddagger}$.

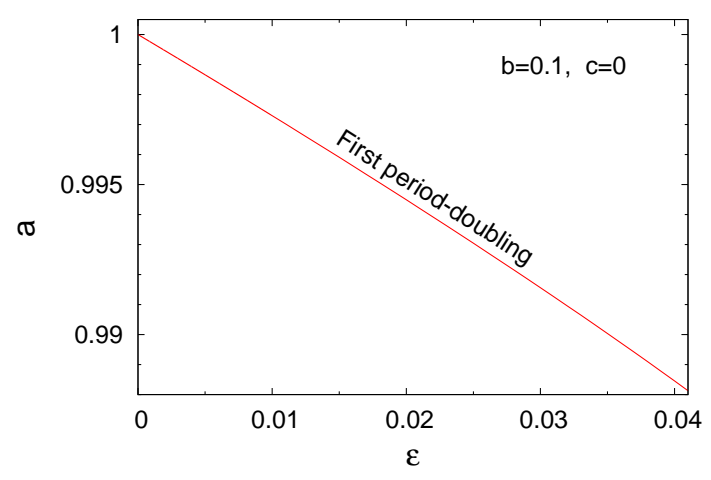

Figure 7: (Color Online). Curve of the first period-doubling bifurcation.

At $\varepsilon=0$ the equations of motion are

$$
\begin{aligned}
d X / d \tau & =X^{2}-U \\
d U / d \tau & =2 X \\
d V / d \tau & =0
\end{aligned}
$$

\footnotetext{
${ }^{\ddagger}$ Note that the bracketed expression coincides with the $\varepsilon$-independent part of the expansion (2.3) for $a_{H}$.
} 
Besides an obvious integral of motion $V$, equations (4.3) possess another integral: $I=\left(X^{2}-\right.$ $U-1) \mathrm{e}^{-U} ;-1 \leq I<+\infty$. In the 3-dimensional phase space, all trajectories of (4.3) are intersections of integral planes $V=$ const with integral surfaces $I=$ const. For $-1 \leq I<0$ trajectories are closed curves which correspond to periodic motions; for $I \geq 0$ the motions are unbounded.

On a secant plane transversal to the set of periodic orbits, the two-dimensional Poincaré mapping becomes an identity, and, therefore, is area-preserving; this confirms the above conjecture on the conservative character of dynamics in the limit $\varepsilon \rightarrow 0$. Inside the continuum of periodic orbits, every perturbation is neutral. Therefore both Poincaré multipliers of each periodic orbit equal 1 . As soon as a non-zero value of $\varepsilon$ is introduced, the integrals break up, only isolated periodic solutions can survive, and chaos can appear. With respect to the system (4.3), equations (4.2) pose a singular perturbation: presence of period-doubling bifurcations at arbitrarily small values of $\varepsilon$ implies the abrupt change of the Poincaré multiplier. Numerical integration shows that at small values of $\varepsilon$ the periodic solutions of (4.2) stay close to the unperturbed integral curves of (4.3) which correspond to small values of $I$. In Fig.8 temporal evolution of $I$ and $V$ along the periodic solution of (4.2) is presented. Variation of both "integrals" over the period is of the order of $\sqrt{\varepsilon}$. Noteworthy, $V(\tau)$ exhibits the nearly harmonic oscillation, whereas the value of $I(\tau)$ stays, save for two relatively short excursions, nearly constant in a narrow strip of the width $\sim \varepsilon$.
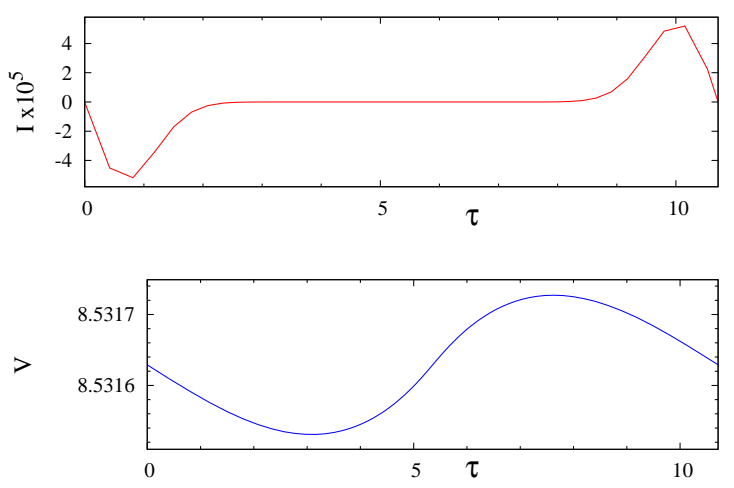

Figure 8: (Color Online). Evolution of perturbed "integrals" along the periodic orbit. $\varepsilon=10^{-10}$, $b=0, c=1, a=0.9999999997492093(A=-5.01581443 \ldots)$.

\section{Summary and Discussion}

In this study we have investigated a simplistic model in which the membrane potential of a neuron interacts with two gating variables. The model includes one fast variable and two slow ones. Under variation of parameter, the system exhibits transition from quiescent state to the regime of periodic spiking. The intermediate stages include the sequence of period-doubling bifurcations for 
subthreshold oscillations, and the mixed-mode oscillations with intermittent spikes. In the limit of the vanishing coefficient of timescale separation, dynamics is conservative.

To my knowledge, this is the first example of such dynamics in the minimalistic model of a single neuron. Aperiodic state for these equations, observed in [12], corresponds to the different parameter region without a clear separation into one fast and two slow variables. The perioddoubling sequence in [7] was detected near the codimension-three instability (triple zero eigenvalue), - again far away from the case of a single equilibrium state considered above; there neither the amplitudes of oscillations nor the timescales are separated. As shown in [7], the resulting chaotic state was equivalent to the well-known Rössler chaotic attractor [13], and dynamics upon it showed no alteration of fast and slow stages.

\section{Acknowledgments}

My research was supported by SFB-555 from the Deutsche Forschungsgemeinschaft. Discussions with L. Schimansky-Geier, K. Schneider, J. Sneyd, D. Turaev are gratefully acknowledged.

\section{References}

[1] V. I. Arnold (Editor). Dynamical systems V: Bifurcation theory and catastrophe theory. Encyclopaedia of Mathematical Sciences. Springer. New York, Berlin, Heidelberg, 1999.

[2] M. Brons, M. Krupa, M. Wechselberger. Mixed mode oscillations due to the generalized canard phenomenon. Fields Institute Communications, 49 (2006), 39-63.

[3] M. Brons, T. J. Kaper, H. G. Rotstein (Editors). Mixed Mode Oscillations: Experiment, Computation, and Analysis. Focus Issue of Chaos, 18 (2008).

[4] J. L. Callot, F. Diener, M. Diener. Problem of duck hunt. Compt. Rend. Acad. Sci., 286 (1978), 1059-1061.

[5] P. Collet, J.-P. Eckmann, H. Koch. On universality for area-preserving maps of the plane. Physica D, 3 (1981), 457-467.

[6] W. Eckhaus. Relaxation oscillations including a standard chase on French ducks. Lect. Notes Math., 985 (1983), 449-494.

[7] G. B. Ermentrout. Period doublings and possible chaos in neural models. SIAM J. Appl. Math., 44 (1984), 80-95.

[8] M. J. Feigenbaum. Quantitative universality for a class of nonlinear transformations. J. Stat. Phys., 19 (1978), 25-52.

[9] J. M. Greene, R. S. MacKay, F. Vivaldi, M. J. Feigenbaum. Universal behaviour in families of area-preserving maps. Physica D, 3 (1981), 468-486. 
[10] J. Keener, J. Sneyd. Mathematical physiology. Springer, New York, 1998.

[11] A. Milik, P. Szmolyan, H. Löffelmann, E. Gröller. The geometry of mixed-mode oscillations in the 3d-autocatalator. Int. J. Bif. \& Chaos, 8 (1998), 505-519.

[12] J. Rinzel. Formal Classification of bursting mechanisms in excitable systems. Lecture Notes Biomathematics, 71 (1987) 267-281, Springer, New York.

[13] O. E. Rössler. An equation for continuous chaos. Phys. Lett. A, 57 (1976), 397-398 .

[14] H. G. Rotstein, R. Kuske. Localized and asynchronous patterns via canards in coupled calcium oscillators. Physica D, 215 (2006), 46-61.

[15] X. Sailer, M. Zaks, L. Schimansky-Geier. Collective dynamics in an ensemble of globally coupled FHN systems. Fluctuation \& Noise Lett., 5 (2005), L299-L304.

[16] T. Verechtchaguina, I. M. Sokolov, L. Schimansky-Geier. First passage time densities in nonMarkovian models with subthreshold oscillations. Europhys. Lett., 73 (2006), 691-697.

[17] M. Wechselberger. Existence and bifurcation of canards in $R^{3}$ in the case of a folded node. SIAM J. Appl. Dyn. Sys., 4 (2005), 101-139.

[18] M. A. Zaks, X. Sailer, L. Schimansky-Geier, A. Neiman, Noise induced complexity: from subthreshold oscillations to spiking in coupled excitable systems. Chaos, 15 (2005), 026117 $1-13$.

[19] A. B. Zisook. Universal effects of dissipation in two-dimensional mappings. Phys. Rev. A, 24 (1981), 1640-1642. 